\title{
Biopsy diagnosis of CD30-positive anaplastic large T-cell lymphoma of ileum in necrotic biopsies
}

\author{
Tadashi Terada* \\ Department of Pathology, Shizuoka City Shimizu Hospital, Shizuoka, Japan
}

Received: December 29, 2015

Accepted: February 25, 2016

Online Published: March 15, 2016

DOI: $10.5430 /$ crep.v3n2p43

URL: http://dx.doi.org/10.5430/crcp.v3n2p43

\begin{abstract}
CD30 (Ki-1)-positive anaplastic large cell lymphoma (ALCL) has been reported only once in ileum. A 72-year-old presented with melena for 3 weeks duration. Endoscopy identified a large ulcerated tumor $(3 \mathrm{~cm} \times 4 \mathrm{~cm} \times 4 \mathrm{~cm})$ in ileum proximal to Bauhin's valve. Punch biopsies showed broad coagulative necrosis without apparently viable cells, but cell contours were recognizable. Diagnosis was impossible on HE-section, but it suggested malignancy. Since the patient denied second endoscopy, the author managed to make the diagnosis of necrotic punches. Immunohistocheimcally, the coagulative necrosis cells were positive for vimentin, CD45, CD3, CD4, CD5, CD10, CD15, CD30, CD43, CD45RO, p53, and Ki-67 (labeling = 78\%). They were negative for cytokeratin (CK) AE 1/3, CK CAM5.2, CK5, CK6, CK7, CK8, CK18, CK19, CK20, EMA, p63, CD8, CD20, CD38, CD138, CD23, CD79 $\alpha, \kappa$-chain, $\lambda$-chain, bcl-2, bcl-6, cyclinD1, CD56, CD57, TdT, chromogranin, synaptophysin, NSE, S100 protein, smooth muscle actin, MUC1, MUC2, MUC5AC, MUC6, KIT and PDGFRA. Since CD30 and CD15 were strongly positive, Hodgkin's disease and ALCL were considered. However, Hodgkin's disease was unlikely because no apparent Hodgkin's or Reed-Sternberg cells were seen. Therefore, CD30-positive ALCL was diagnosed. In addition, because the tumor cells expressed only T-cell markers, the diagnosis of CD30-positive T-cell ALCL was confirmed. The present study could not detect ALK, ALK/NPN, ALK-1, p80, and tissue destructive molecules such as perforins, TIA-1, and granzyme B because tumor cells were totally necrotic. Chromosomal translocation of $\mathrm{t}(2 ; 5)$ and TCR rearrangement could not be performed due to no viable cells. Post-diagnosis whole body examinations using CT, MRI, and PET showed bladder metastasis and multiple lymph node swellings in the para-aortic areas (Ann Arber Stage 4). The patient is now treated by chemotherapy and radiation for 5 months. The present study showed a very rare case of ileal CD30-positive T-cell ALCL and indicated that lymphoma diagnosis is possible even in coagulatively necrotic tissues.
\end{abstract}

Key Words: CD30, Anaplastic large cell lymphoma, Ileum, Biopsy, Necrosis, T-cell neoplasms

\section{INTRODUCTION}

CD30-positive anaplastic large cell lymphoma (ALCL) had been termed Ki-1 lymphoma. However, in recent years, it has been called CD30-positive ALCL. Most of them are of T-cell phenotype, and B-cell, NK/T-cell ALC are very rare. In recent WHO blue, the name ALCL was confined to Tcell neoplasms, and CD30-positive B-cell neoplasms with anaplasia are classified into diffuse large B-cell lymphoma (DLBCL).

The histopathological and clinical features of CD30-positive ALCL were changed by the discovery of anaplastic lymphoma kinase (ALK) which is associated with chromosomal translocation of $\mathrm{t}(2 ; 5)$ and a resultant fusion gene of ALK.

\footnotetext{
*Correspondence: Tadashi Terada; Email: piyo0111jp@yahoo.co.jp; Address: Department of Pathology, Shizuoka City Shimizu Hospital, Miyakami 1231 Shimizu-Ku, Shizuoka 424-8636, Japan.
}

Published by Sciedu Press 
This discovery drastically changed the treatment and classification of ALCL. Patients with ALK-positive ALCL tended to be young (median age $=25$ years) and are high responder for brentuximab vedotin. The prognosis is good if this drug is used. However, patient with ALK-negative CD30-positive ALCL shows poor prognosis. Pathologically, CD30-positive ALCL tends to be positive for EMA, granzyme B, TIA-1. In general CD15 and Epstein-Barr virus (EBV) related antigens are not detected in ALK-positive ALCL. ${ }^{[1-7]}$ In general, ALK-negative ALCL affects older persons (median $=57$ years), and when discovered the disease is advanced stage. ALK-negative ATCL showed much more pleomorphic than ALK-positive ATCL. This type of ALCL may shows CD15 and EBV positivity.

Almost all lymphomas of the gastrointestinal tracts are of B-cell type. ALCL in small intestine is extremely rare. Only one case of ALCL in ileum has been reported. ${ }^{[8]}$ Herein reported is an extremely rare case of primary Ileal CD30positive ALCL. The diagnosis was made in biopsies that showed only coagulative necrosis.

\section{CASE REPort}

A 72-year-old man presented melena of 3 weeks duration. Physical examination showed mild fever $\left(37.6^{\circ} \mathrm{C}\right)$ and anemia. A blood laboratory data revealed diabetes mellitus (HbA1c; 7.2\%). The tumor markers (CEA and CA19-9) were within normal ranges. Colon fiberscopy showed abnormal findings of ileum. Thus, double balloon small intestinal fiberscope was done, detecting a large ulcerated tumor $(3 \mathrm{~cm} \times 4 \mathrm{~cm} \times 4 \mathrm{~cm})$ in ileum $70 \mathrm{~cm}$ proximal to Bauhin's valve (see Figure 1). Five pinch biopsies were taken. No aspiration biopsy, US-guided biopsy, or aspiration biopsy cytology were carried out.

All the 5 punches of showed necrotic tissue (see Figure 2A). The necrosis was coagulative one, and cell contour was recognizable in most areas (see Figure 2B). No apparent Hodgkin's or Reed-Sternberg cells were seen. The diagnosis was impossible on HE-section, but it suggested malignancy because of massive necrosis (see Figures 2A-B). Since the patient denied the second fiberscope, the author tried the diagnosis of the necrotic 5 punches.

An immunohistochemical study was done with the use of Dako-Envision method as previously reported. ${ }^{[9]}$ Immunohistocheimcally, the coagulative necrosis cells were positive for vimentin, CD45, CD3 (see Figure 3A), CD4 (see Figure 3B), CD5, CD10, CD15 (see Figure 3C), CD30 (see Figure 3D), CD43, CD45RO, p53 (see Figure 3E), and Ki67 (labeling=78\%) (see Figure 3F), but were negative for cytokeratin (CK) AE 1/3, CK CAM5.2, CK5, CK6, CK7,
CK8, CK18, CK19, CK20, EMA, p63, CD8, CD20, CD38, CD138, CD23, CD79 $\alpha, \kappa$-chain, $\lambda$-chain, bcl-2, bcl-6, cyclinD1, CD56, CD57, TdT, chromogranin, synaptophysin, NSE, S100 protein, smooth muscle actin, MUC1, MUC2, MUC5AC, MUC6, KIT and PDGFRA. Since both CD30 and CD15 were strongly positive, the diagnosis of CD30positive ALCL-related lymphoma was made. In addition, because the tumor cells expressed only T-cell markers, sparing antigens of B-cells and NK/T cells, lymphoblastic cells, the diagnosis of CD30-positive T-cell ALCL was made. The present study could not detect ALK/NPN, ALK-1, p80, and tissue destructive molecules such as perforins, TIA-1, and granzyme B because the tumor necrosis were marked. Chromosomal translocation of $\mathrm{t}(2 ; 5)$ and TCR rearrangements also could not be performed due to no viable cells. Therefore, it was not determined whether the tumor is ALK-positive or ALK-negative T-cell CD30 positive ALCL.

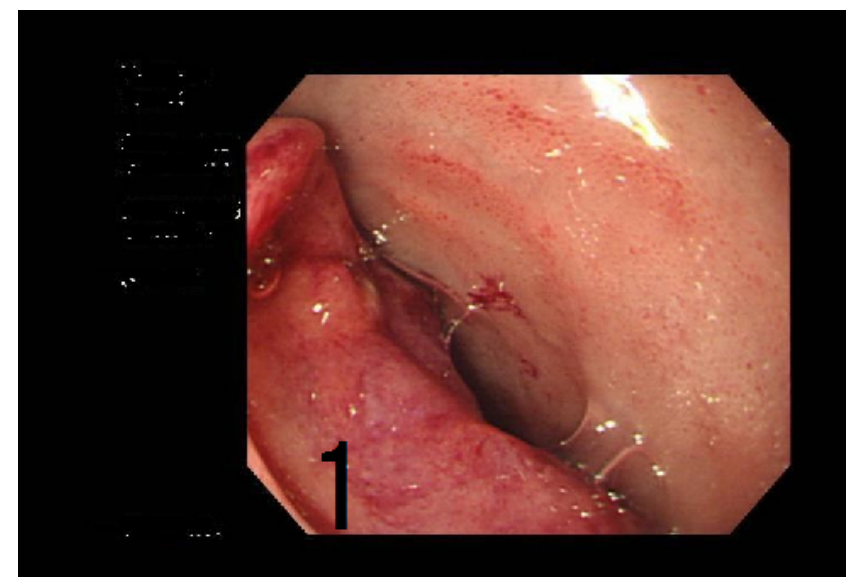

Figure 1. Ileum fiberscope revealed a large ulcerated tumor

Post-diagnosis whole body examination using CT, MRI, and PET showed bladder metastasis and multiple lymph node swelling in para-aortic regions. The patient is now alive and is treated by chemotherapy (CHOP) and radiation for 5 months with mild decrease of tumors' volume.

\section{DiscuSSION}

Most of gastrointestinal malignant lymphoma are B-cell neoplasms. ${ }^{[10]}$ T-cell malignant lymphoma of gastrointestinal tract is extremely rare. The most common occurrence site of malignant lymphoma of gastrointestinal tract is stomach, where MALT lymphoma is most prevalent. Malignant lymphoma in small intestine is very rare. In addition, T-cell malignant lymphoma of small intestine is extremely rare. Further, CD30-positive ALCL of ileum is extremely rare; only one case report has been present. ${ }^{[1]}$ The present case is the second case of ileal CD30-positive T-cell ALCL. 


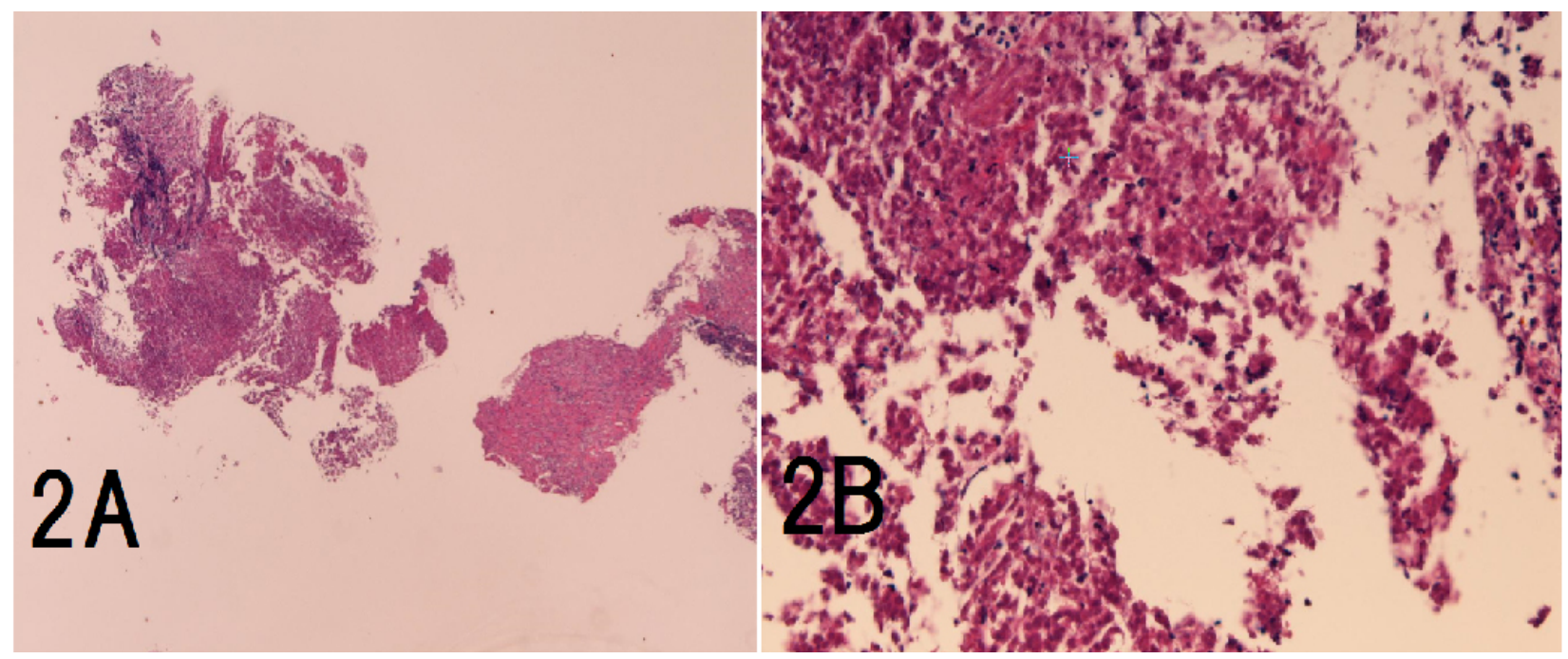

Figure 2. The biopsy specimens

The biopsy shows only colagulative necrotic tissue. The contours of the necrotic cells are seen, but no cell details are seen. HE staining. A: Low power view. B: High power view. A: $\times 20 . B: \times 200$.
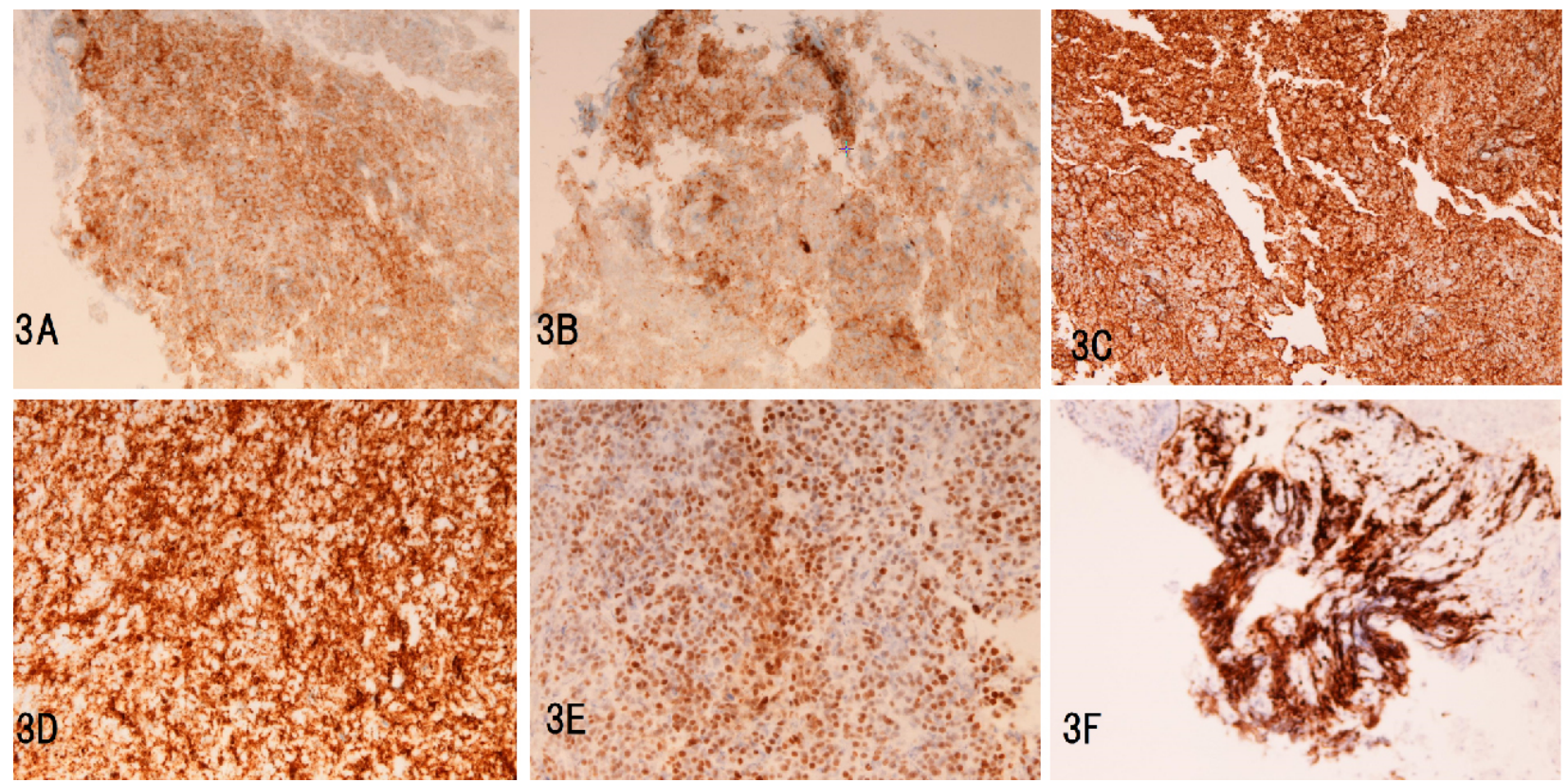

Figure 3. Immunohistochemical findings

The cells of coagulative necrosis cells are positive for CD3 (A), CD4 (B), CD15 (C), CD30 (D), p53 (E), and Ki-67 (labeling $=78 \%)(F)$. A-F: immunostainings, $\times 200$

The pathological diagnosis of ALCL seems valid in the present case. Cell histologies and cellular details could not be seen because tumor cells showed coagulative necrosis. However, the tumor cells showed leukocytic antigen CD45 and were negative for epithelial antigen CKs. The author considered small cell carcinoma and malignant lymphoma on the HE preparation, because small cell carcinoma and malignant lymphoma were very similar in morphological features on HE sections in ill-fixed and necrobiotic spec- imens. Small cell carcinoma could be completely denied because of negative CK and negative CD56 (NCAM), NSE, synaptophysin and chromogranin. Because the tumor cells were positive for T-cell markers and were negative for B-cell antigens, NK-cell antigens (CD56, CD57), and plasma cell antigens (CD38, CD79 $\alpha$, and CD138), the tumor is certainly T-cell lymphoma. The malignant potential was confirmed by strongly positive p53 and very high Ki-67 labeling. The positive p53 suggests positive p53 mutations and also suggest that 
the molecular genesis of the present T-ALCL involves p53 signal transduction. The present case showed CD4+/CD8pattern, suggesting tumor cells are of helper/inducer characters, similar to cutaneous mycosis fungoides.

The present T-cell malignant was strongly positive for CD30 and CD15. CD30 and CD15 are expressed in Hodgkin's disease and ALCL. However, no apparent Hodgkin's or Reed-Sternberg cells were seen, although the tissue was necrotic. Thus, the present tumor is not Hodgkin's disease. The monotonous features of the tumor cell contours are also against the diagnosis of Hodgkin's disease. Since CD30 and CD15 were strongly expressed and only T-cell antigens were expressed in the current tumor, the diagnosis of CD30positive ALCL in the present case seems correct.

CD30-positive ALCL is now classified into ALK-positive and ALK-negative ALCL. Both categories show significantly different clinical and pathological aspects as well as therapeutic regiments. Unfortunately, the classification with regard to ALK could not be performed, because immunostains of ALK, granzyme B, TIA-1 and perfolin failed or were negative in the tumor. Chromosomal translocation of $\mathrm{t}(2 ; 5)$ and TCR rearrangements also could not be performed due to no viable cells in the biopsy. In addition, the status of ALK, which can determine therapeutic strategy, was unclear

\section{REFERENCES}

[1] Stein H, Foss HD, Durkop H, et al. CD30-positive anaplastic large cell lymphoma: a review of its histopathologic, genetic, and clinical features. Blood. 2000; 96: 3681-95. PMid: 11090048.

[2] Jaffe ES. Anaplastic large cell lymphoma: the shifting sands of diagnostic hematopathology. Mod Pathol. 2001; 14: 219-28. PMid: 11266530. http://dx.doi.org/10.1038/modpathol.3 880289

[3] Morris SW. ALK+ CD30+ lymphomas: a distinct molecular genetic subtype of non-Hodgkin's lymphoma. Br J Haematol. 2001; 113: 275 95. http://dx.doi.org/10.1046/j.1365-2141.2001.02574 . $\mathrm{x}$

[4] Delsol G. Anaplastic large cell lymphoma. In: Jaffe ES, Harris NL, Stein H, Vardiman JW, eds. World Health Organization Classifications of Tumors: Tumours of Haematopoietic and Lymphoid Tissues. IARC Press, Lyon, 230-35, 2001.

[5] Touriol C, Grennland C, Lamant L, et al. Further demonstration of the diversity of chromosomal changes involving 2p23 in ALK-positive lymphoma: 2 cases expressing ALK kinase fused to CLTCL (clathrin chain polypeptide-like). Blood. 2000; 95: 3204-7. PMid: 10807789.

[6] Suzuki R, Kagami Y. Takeuchi K, et al. Prognostic significance of CD56 expression for ALK-positive and -negative anaplastic large also because the patient denied the second small intestinal balloon endoscopy that is aggressive endoscopy.

The author thought on the HE staining that the necrotic tissue may be malignancy but did not think that correct diagnosis would be made by immunohistochemistry. Because the patient refused second endoscopy, the author was forced to diagnose the first necrotic biopsy. The author performed many immunostainings, and surprised to find that the diagnosis was possible only in immunohistochemical stainings; it means that some cases express antigenicity of tumor cells even if they become coagulatively necrotic. Thus, pathologists should performed immunohistochemical study even if tumor is totally necrotic. The cell death can be apoptosis, which can be detected by in situ nick endolabelling or agarose gel electrophoresis after DNA extraction. ${ }^{[11]}$

\section{Conclusion}

The author reported an extremely rare case of CD30-positive T-cell ALCL. Of interest, the diagnosis was possible in punch biopsies showing total coagulative necrosis of tumor cells. However, the immunohistochemistry could not identify ALK and tissue destructive molecules in my case.

\section{CONFLicts OF InTEREST Disclosure}

The author has no conflict of interest. cell lymphoma of T/null cell phenotype. Blood. 2000; 96: 2993-3000. PMid: 11049976

[7] Jaffe ES, Harris NL, Stein H, et al. World Health Organization Classification of Tumors. Pathology and Genetics of Tumours of Hematopietic and Lymphoid Tissues. IARC Press, Lyon, 2001.

[8] Cooperberg MR, Fiedler PN. Ki-1 anaplastic large-cell lymphoma occurring at the site of ileocolonic anastomosis in a patient treated surgically for colonic adenocarcinoma: case report and review of the literature. Ann Diagn Pathol. 2001; 5: 162-7. PMid: 11436170. http://dx.doi.org/10.1053/adpa.2001.25408

[9] Terada T, Kawaguchi M, Furukawa K, et al. Minute mixed ductalendocrine carcinoma of the pancreas with predominant intraductal growth. Pathol Int. 2002; 52: 740-6. PMid: 12685552. http: //dx.doi.org/10.1046/j.1440-1827.2002.01416.x

[10] Terada T. Gastrointestinal malignant lymphoma: a pathologic study of 37 cases in a single Japanese institution. Am J Blood Res. 2012; 2: 194-200. PMid: 23119230.

[11] Terada T, Nakanuma Y. Detection of apoptosis and expression of apoptosis-related proteins during human intrahepatic bile duct development. Am J Pathol. 1995; 146: 67-74. PMid: 7531950. 